\section{Efeitos de exercícios resistidos, de equilíbrio e alongamentos sobre a mobilidade funcional de idosas com baixa massa óssea}

\author{
Effects of resistance, balance and stretching \\ on functional mobility in elderly with low \\ bone mass
}

\author{
Mariana de Sousa Moura ${ }^{1}$ \\ Márcia Alessandra Carneiro Pedrosa ${ }^{1}$ \\ Eduarda Lubambo Costa ${ }^{1}$ \\ Paulo Sérgio de Castro Bastos Filho ${ }^{1}$ \\ Larissa Bouwman Sayão ${ }^{1}$ \\ Tiago Siqueira de Sousa ${ }^{1}$
}

\begin{abstract}
Resumo
O envelhecimento é caracterizado por degeneração da função neuromuscular traduzida por diminuição da força muscular, do equilíbrio e da coordenação. Este estudo visou avaliar os efeitos de programas de exercícios resistidos, de equilíbrio e de alongamentos sobre a mobilidade funcional em idosas com baixa massa óssea. 36 voluntárias foram aleatorizadas em dois grupos: $\mathrm{G} 1$ [n=18; $69,4 \pm 6,8$ anos] e G2 [n=18; 69,4 \pm 5,7 anos] e avaliadas através do Teste Sentar-levantar com 5 repetições (STS-5), Teste de Caminhada de seis metros (SMW) e Teste de Alcance funcional (FRT). O programa de exercício foi aplicado durante 4 meses, 2 vezes por semana, com treinamento de força muscular e mobilidade funcional para G1, e alongamentos e palestras informativas para G2. Após a intervenção os indivíduos de ambos os grupos diminuíram o tempo de realização do STS-5 (G1= 12,6 $\pm 3,5$ vs. $8,4 \pm 1,3 \mathrm{~s}, \mathrm{p}=0,01 ; \mathrm{G} 2=12,2 \pm 2,6$ vs. $8,1 \pm 1,3 \mathrm{~s}, \mathrm{p}=0,00)$, bem como do SMW (G1=4,4 $\pm 1,2$ vs. $3,9 \pm 0,8$ s, $p=0,014 ; G 2=4,2 \pm 0,5$ vs. $3,6 \pm 0,4 s, p=0,000$ ), enquanto se evidenciou aumento do alcance no FRT (G1=26,4 $\pm 4,9$ vs. $31,6 \pm 5,0 \mathrm{~cm}, \mathrm{p}=0,001 ; \mathrm{G} 2=24,6 \pm 4,8$ vs. $30,3 \pm 4,3 \mathrm{~cm}, \mathrm{p}=0,001)$. Os nossos resultados permitem concluir que foram encontradas melhoras em ambos os grupos estudados e que os exercícios físicos propostos são capazes de trazer benefícios para idosas com baixa massa óssea, através de melhora na sua mobilidade funcional.
\end{abstract}

\section{Palavras-chave}

Idoso; Exercício; Osteoporose; Acidentes por Quedas; Treinamento de Resistência; Equilíbrio Postural.

\begin{abstract}
Aging is characterized by neuromuscular function degeneration reflected on a decrease of muscle strength, balance and coordination. This study aimed to evaluate the effects of resistance, balance and stretching exercise programs on functional mobility in community-dwelling elderly with low bone mass. 36 volunteers were randomized into two groups: $G 1$ [ $n=18 ; 69.4 \pm 6.8$ years] e G2 [n=18; 69.4 \pm 5.7 years] which were evaluated using the Sit-to-stand-5 Test (STS-5), Six metre walk test (SMW) and Functional Reach Test (FRT). The exercise program was applied for 4 months, two times per week, with muscle strength and functional mobility training for G1, and stretching and informative lectures for G2. After the intervention, individuals from both groups decreased their time of performance in STS-5 (G1=12.6 \pm 3.5 vs. $8.4 \pm 1.3 \mathrm{~s}, p=0.01$; $G 2=12.2 \pm 2.6$ vs. $8.1 \pm 1.3 \mathrm{~s}, p=0.00$ ), as well as in $S M W(G 1=4.4 \pm 1.2$ vs. $3.9 \pm 0.8 s, p=0.014 ; G 2=4.2 \pm 0.5$ vs. $3.6 \pm 0.4 s, p=0,000)$, while an increase in the reach of FRT was evident $(G 1=26.4 \pm 4.9$ vs. $31.6 \pm 5.0 \mathrm{~cm}, p=0.001$; $G 2=24.6 \pm 4.8$ vs. $30.3 \pm 4.3 \mathrm{~cm}, p=0.001$ ). The results indicate that improvements were found in both studied groups and that the proposed exercise programs are able to provide benefits for elderly with low bone mass, throughout the improvement in their functional mobility.
\end{abstract}

\section{Keywords}

Aged. Exercise. Osteoporosis. Accidental Falls. Resistance Training. Postural Balance.
Rev Bras Ativ Fis Saúde p. 474-484 DOI: http://dx.doi.org/10.12820/23171634.2012v17n6p474

1 Departamento de Fisioterapia, Universidade Federal de Pernambuco, Recife, PE, Brasil 


\section{INTRODUÇÃO}

O envelhecimento populacional é um fenômeno que ocorre em todo o mundo. Tal fato é relevante para os custos econômicos com a saúde da população, pois acarretará em um grande aumento do número $\left(\mathrm{n}^{\circ}\right)$ de quedas, casos de osteoporose e de fraturas osteoporóticas (FO). No Brasil estima-se que, em 2009, o SUS tenha gasto em torno de $\mathrm{R} \$ 81$ milhões em tratamentos de fraturas em idosos ${ }^{1}$.

Segundo a Organização Mundial de Saúde, osteoporose (OP) é uma doença caracterizada por diminuição da massa óssea, deterioração da microarquitetura do tecido ósseo e aumento da fragilidade mecânica, tornando o osso predisposto a fraturas por traumas mínimos ${ }^{2}$. Até a ocorrência de fraturas, esta doença não apresenta sintomas.

Além disso, indivíduos com OP apresentam redução da função neuromuscular traduzida por diminuição da força e potência muscular, do equilíbrio estático e dinâmico, bem como da coordenação ${ }^{3,4,5}$. O envelhecimento promove ainda redução da sensibilidade proprioceptiva dos membros inferiores e a transmissão mais lenta das informações responsáveis por manter o equilíbrio, o que contribui de modo significativo para o aumento do $n^{\circ}$ de quedas $s^{6,7}$. A principal consequência da queda é a fratura, que pode levar os idosos à incapacidade, necessidade de institucionalização e reabilitação, internamento ou mesmo ao óbito, gerando um alto custo social e redução da qualidade de vida ${ }^{3,8,9}$.

Além do maior $\mathrm{n}^{\circ}$ de quedas, o declínio da capacidade funcional em idosos leva à redução das funções físicas e cognitivas ${ }^{9}$ associada à mobilidade prejudicada, causando incapacidade e dependência na realização de atividades de vida diária ${ }^{5}$. Assim, considera-se importante avaliar o declínio na mobilidade funcional em idosos, que muitas vezes se demonstra pela lentidão na velocidade da marcha ${ }^{5}$. Podem ser citados como fatores que influenciam a mobilidade: sistema nervoso central e periférico, sistema sensorial, produção de energia, músculos, ossos e articulações ${ }^{9}$. De fato, existe uma associação entre força muscular, equilíbrio e mobilidade funcional, tanto que segundo um estudo de Granacher et al , indivíduos que realizaram fortalecimento de musculatura estabilizadora do tronco obtiveram melhoras na sua mobilidade funcional.

O exercício é uma das estratégias mais importantes para manutenção da DMO e prevenção da $\mathrm{OP}^{3,10}$. Além disso, vários trabalhos ratificam os efeitos benéficos sobre a força muscular, coordenação e mobilidade funcional $1^{7,11,12,13}$, proporcionando redução do $\mathrm{n}^{\circ}$ de quedas em idosos. No entanto, em recente estudo de revisão sistemática foi identificado que nem todos os protocolos de exercícios promovem bons resultados, e que exercícios para indivíduos com baixa massa óssea devem adotar atividades aeróbicas e de equilíbrio, exercícios com ou sem fortalecimento muscular, com e sem controle de peso ${ }^{14}$. De fato, em um estudo de revisão sistemática, Orr et $a l^{15}$ analisaram a eficiência do programa de treinamento resistido na melhora do equilíbrio em idosos, identificando 29 estudos e 68 testes para avaliação de equilíbrio, dos quais apenas $22 \%$ dos estudos relataram benefícios. Os autores atribuíram tal resultado à heterogeneidade dos testes e falhas de metodologia.

Portanto, mais estudos são necessários para avaliar a influência de diferentes tipos de exercícios na promoção do controle postural e da musculatura relacionada ao equilíbrio ${ }^{4}$. Em vista disso, este estudo avaliou os efeitos de programas de exercícios resistidos, de equilíbrio e alongamentos sobre a mobilidade funcional em idosos com baixa massa óssea. 


\section{MÉTODOS}

\section{Amostra}

Após a pesquisa ter sido aprovada pelo Comitê de Ética em Pesquisa do Centro de Ciências da Saúde da UFPE (CAEE 0359.0.172.000-08), foram recrutados pacientes atendidos no ambulatório de Reumatologia Clínica do Hospital das Clínicas (HC) e na disciplina de Reumatofisioterapia do curso de Fisioterapia da UFPE.

Para a inclusão dos participantes foram usados os seguintes critérios: indivíduos de ambos os sexos, com idade igual ou superior a 60 anos, capazes de entender e de responder aos comandos utilizados durante os testes de mobilidade funcional, e capazes de deambular sem assistência ou supervisão de outros. Os indivíduos deveriam apresentar diagnóstico de baixa massa óssea - osteopenia ou osteoporose, definidos respectivamente, conforme critério da $\mathrm{OMS}^{2}$, por um T-score de -1 a -2,4 desvio padrão (DP) e por um T-score -2,5 DP abaixo da média para mulheres brancas com idade entre 20 e 30 anos a partir do exame de densitometria óssea por raios X de dupla energia (DXA), em nível da coluna lombar L1-L4, colo femoral ou fêmur total.

Foram selecionados 61 indivíduos para uma avaliação de história clínica e testes funcionais, mencionados no fluxograma descrito na Figura 1. Entretanto, 25 desses foram excluídos por corresponderem a algum dos critérios de exclusão, como: praticar atividade física regularmente duas vezes por semana ou mais; história de doença ou uso de medicamentos que prejudiquem o equilíbrio e o metabolismo ósseo (ex.: vestibulopatias, doença de Parkinson, hiperparatiroidismo primário); hipertensão arterial não controlada (sistólica > $200 \mathrm{mmHg}$ e/ou diastólica $>100 \mathrm{mmHg}$ ); presença de condição reumatológica que impossibilitasse a realização ativa dos exercícios; dependência de álcool e drogas ilícitas.

Os participantes selecionados foram submetidos à avaliação, que se constituiu de anamnese, exame físico e testes de função neuromuscular.

\section{Anamnese e Exame Físico}

Foram coletados dados de: identificação pessoal; história clínica (hipertensão, diabetes, complicações cardíacas, alterações neurológicas e outras alterações reumatológicas); história familiar; ingestão de cálcio complementar, alimentos ricos em cálcio e exposição solar; tabagismo; atividade física; limitações nas atividades de vida diária (AVDs); medicações em uso e história de quedas e fraturas. No exame físico foram aferidos os sinais vitais (pressão arterial, frequência cardíaca e respiratória), mensurados a altura e o peso, e calculado o IMC.

Considerando que as quedas podem estar associadas às disfunções cognitivas ${ }^{16}$ foi importante assegurar a capacidade de entender os comandos durante a avaliação e as sessões de terapia, os voluntários foram submetidos ao mini exame do estado mental (MEM) ${ }^{17}$.

\section{Testes de Mobilidade Funcional}

A avaliação dos parâmetros de função neuromuscular foi realizada através dos seguintes testes de mobilidade funcional: teste de sentar-levantar com 5 repetições- Sit-to-stand test, o teste de caminhada de 6 metros- Six-metre-walk e o teste de alcance funcional - Functional Reach Test. Tais testes já foram elucidados como fatores de predição na mobilidade funcional e risco de quedas de idosos ${ }^{18,19}$, justificando assim, a sua utilização para avaliar o desempenho de um grupo de idosos com osteoporose. 
Os testes foram realizados duas vezes por cada participante, de forma que o melhor resultado foi selecionado para análise. $\mathrm{O}$ mesmo avaliador realizou os mesmos testes, antes e após a intervenção, em ambos os grupos investigados. Cinco dos voluntários previamente selecionados não conseguiram realizar todos os testes funcionais propostos, sendo assim excluídos da pesquisa (Figura 1).

\section{Teste de sentar-levantar com 5 repetições: Sit-to-stand test (STS-5)}

Os indivíduos foram solicitados a se levantar de uma cadeira sem braços com $43 \mathrm{~cm}$ de altura, 5 vezes seguidas, o mais rápido que conseguissem mantendo os braços cruzados à frente do tronco. $\mathrm{O}$ tempo em segundos foi medido desde a posição inicial sentada até a posição final sentada, depois de completadas as 5 repetições ${ }^{18}$.

\section{Teste de caminhada de 6 metros: Six-metre-walk test (SMW)}

Foi mensurado o tempo que o participante percorria $6 \mathrm{~m}$ caminhando o mais rápido possível. Para assegurar que a velocidade da marcha fosse mantida constante, foram acrescentadas as distâncias de 2 metros antes e 3 metros após a marcação ${ }^{18}$.

\section{Teste do Alcance Funcional: Functional Reach Test (FRT)}

Foi solicitado que o indivíduo permanecesse de pé com o ombro próximo à parede no ponto inicial de uma fita métrica fixa. $\mathrm{O}$ voluntário foi instruído a realizar uma flexão anterior do braço a $90^{\circ}$ com cotovelo e dedos da mão estendidos para registro do comprimento do membro superior, em seguida solicitou-se uma tentativa de alcançar um objeto à frente com projeção anterior do tronco, sem movimentação dos pés, onde se registrou o ponto de alcance, o resultado foi obtido através do deslocamento horizontal entre a posição inicial e final ${ }^{19}$.

\section{Randomização}

Após a tomada das medidas basais, através de uma randomização simples realizada pelo computador com o programa SPSS (Statistical Package for the Social Sciences) versão 16.0. Os 36 participantes selecionados foram randomizados em dois grupos G1 (Exercícios de fortalecimento muscular e mobilidade funcional); e G2 (Exercícios de alongamento muscular, mobilidade articular e orientações sobre osteoporose) com número equivalente de voluntários. Os dois grupos apresentaram dados homogêneos quanto à idade, condições clínicas, densidade mineral óssea e resultados dos testes de mobilidade funcional (Tabela 1). A amostra reduzida caracterizou tal pesquisa como estudo piloto, a fim de possibilitar embasamento para posteriores intervenções mais abrangentes.

\section{Programa de Exercícios}

A intervenção foi feita duas vezes por semana, no Setor de Fisioterapia do Hospital das Clínicas da Universidade Federal de Pernambuco, tendo cada sessão 50 minutos de duração, durante 4 meses, totalizando 30 sessões. Os dois grupos realizaram suas atividades em horários distintos e sem conhecimento da conduta feita com o outro grupo.

A estrutura da sessão do G1 (Grupo Fortalecimento e Mobilidade Funcional) foi composta por $10 \mathrm{~min}$ de aquecimento, $30 \mathrm{~min}$ de treinamento de força muscular ou treino de equilíbrio e coordenação, seguidos por $10 \mathrm{~min}$ de desaquecimento e relaxamento. $\mathrm{O}$ treino de força e o treino de equilíbrio foram intercalados, de modo que em cada sessão foi realizada apenas uma das formas de treinamento.

Nos 10 min iniciais era realizado o aquecimento através de alongamentos, movimentos de mobilidade articular associados à respiração, breves caminhadas 
e danças circulares. Em seguida durante os 30 min de treinamento de força muscular, foram realizados exercícios de fortalecimento dos músculos do membro inferior e tronco, com maior ênfase nos músculos flexores e extensores do quadril e joelho e estabilizadores do tronco, utilizando pesos livres (halteres e caneleiras) e resistência elástica. Tais como agachamento parcial, mesa extensora e flexora com resistência elástica, panturrilha, abdominais e ponte. Foram realizadas 10 repetições para cada exercício, sendo o número de séries aumentado a cada mês, tendo sido alternadas entre cadeia cinética aberta e fechada. A intensidade do treino variava de acordo com a percepção de esforço relatada pelos indivíduos. Iniciando com posicionamentos em decúbito dorsal, decúbito ventral, e evoluindo para posição sentada e bipedestação ao longo do treinamento.

No treino, para melhorar a mobilidade funcional (equilíbrio e coordenação) foram realizadas atividades em grupo envolvendo movimentos de dança, jogos com bolas e bexigas, treino de marcha com obstáculos, com olhos fechados e com mudanças na velocidade e largura do passo, bem como exercícios de equilíbrio estático com redução progressiva da base de apoio. Também foram realizadas atividades envolvendo subidas e descidas em degraus, com o objetivo de proporcionar algum impacto sobre os ossos dos membros inferiores, e ainda atividades de estímulo proprioceptivo, como locomoção sobre superfícies de diferentes texturas e deslocamento sobre a cama elástica. O desaquecimento ao final de cada sessão era feito através de alongamentos e relaxamento em decúbito dorsal ou na posição sentada.

Já no G2 (Alongamento e Mobilidade Articular), foram realizados alongamentos ativos dos principais grupamentos musculares: cadeia posterior (isquiotibiais, glúteos e musculatura dorsal), trapézio superior, peitoral maior, bíceps braquial, quadríceps, tríceps braquial, tríceps sural, flexores e extensores de punho e tornozelo. Tendo sido utilizadas diversas posições posturais, como decúbito dorsal e ventral, posição sentada e bipedestação em todas as sessões. Foram ainda feitos movimentos de mobilidade articular associados a exercícios respiratórios, de forma que cada participante progredia na intensidade da prática conforme a sua capacidade. Foram utilizados diferentes materiais para auxiliar os exercícios, como bastões, faixas elásticas, bolas e recursos audiovisuais. Realizaram-se ainda, atividades de relaxamento e palestras informativas sobre osteoporose, risco de quedas, orientações domiciliares e outros temas relacionados à saúde.

Todas as atividades foram feitas com o cuidado necessário para evitar fraturas e lesões, respeitando as limitações de cada indivíduo, estando sempre presentes 3 assistentes para orientar corretamente os exercícios dos participantes. O controle da assiduidade era efetuado por um dos pesquisadores em cada sessão, de acordo com a lista de frequência dos participantes.

Após a intervenção, os indivíduos foram submetidos a uma reaplicação da entrevista e dos testes neuromusculares, utilizando os mesmos critérios.

\section{Análise estatística}

Para análise dos dados foi utilizado o software de estatística SPSS versão 16.0 (SPSS Inc, Chicago, IL, EUA). A significância estatística foi considerada se $\mathrm{p}<0,05$. Para a análise estatística dos resultados $(n=36)$ foram utilizados testes paramétricos, pois não houve significância nos testes de normalidade de Kolmogorov-Sminorv e de Shapiro-Wilk, evidenciando uma distribuição normal dos dados. Para comparar os grupos foi utilizado o teste $t$ de Student para amostras não pareadas.

Os resultados obtidos após a intervenção foram analisados através do mesmo software SPSS por meio dos testes t de Student para amostras não pareadas (com- 
paração entre os grupos) e teste t de Student para amostras pareadas (comparação pré e pós no mesmo grupo).

\section{RESULTADOS}

\section{Características Basais dos Grupos de Estudo}

Os 36 voluntários selecionados para a intervenção foram randomicamente separados em dois grupos $\mathrm{G} 1(\mathrm{n}=18)$ e $\mathrm{G} 2(\mathrm{n}=18)$, os quais se mostraram semelhantes entre si $(p>0,05)$ em todos os parâmetros avaliados (Tabela 1$)$.

Tabela 1 - Características basais clínicas e antropométricas do Grupo Fortalecimento e Mobilidade Funcional (G1) e Grupo Alongamento e Mobilidade Articular (G2)

\begin{tabular}{lccc}
\hline Variáveis & $\mathrm{G} 1$ & $\mathrm{G} 2$ & Valor $\mathrm{p}$ \\
\hline Idade (anos) & $69,39(6,76)$ & $69,39(5,73)$ & 1,000 \\
\hline Altura $(\mathrm{cm})$ & $149,30(6,22)$ & $150.30(4,51)$ & 0,630 \\
\hline Peso $(\mathrm{kg})$ & $57,33(11,88)$ & $59,16(12,72)$ & 0,699 \\
\hline Índice de massa corporal & $25,69(4,93)$ & $26,19(5,52)$ & 0,804 \\
\hline Número de quedas & $1,56(1,68)$ & $2,11(1,64)$ & 0,324 \\
\hline Mini-exame do estado mental (escore de 0-30) & $25,33(2,84)$ & $25,78(3,79)$ & 0,694 \\
\hline Número de fraturas & $0,17(0,38)$ & $0,28(0,82)$ & 0,61 \\
\hline DMO colo femoral $\left(\mathrm{g} / \mathrm{cm}^{2}\right)$ & $0,76(0,09)$ & $0,78(0,09)$ & 0,530 \\
\hline DMO trocânter do fêmur $\left(\mathrm{g} / \mathrm{cm}^{2}\right)$ & $0,69(0,08)$ & $0,67(0,09)$ & 0,673 \\
\hline DMO fêmur total $\left(\mathrm{g} / \mathrm{cm}^{2}\right)$ & $0,82(0,13)$ & $0,86(0,09)$ & 0,468 \\
\hline DMO coluna lombar $(\mathrm{L} 1-\mathrm{L} 4)\left(\mathrm{g} / \mathrm{cm}^{2}\right)$ & $0,82(0,13)$ & $0,90(0,16)$ & 0,168 \\
\hline
\end{tabular}

Média, Desvio Padrão (DP) e significância (p). Teste t de Student para amostras não pareadas

Durante a intervenção $(\mathrm{n}=36)$, houve perda amostral devido à desistência dos voluntários, por motivos pessoais como transporte, saúde ou desinteresse (Figura 1). Resultando ao final do estudo num n=27, sendo 13 participantes no $\mathrm{G} 1$ e 14 no $\mathrm{G} 2$.

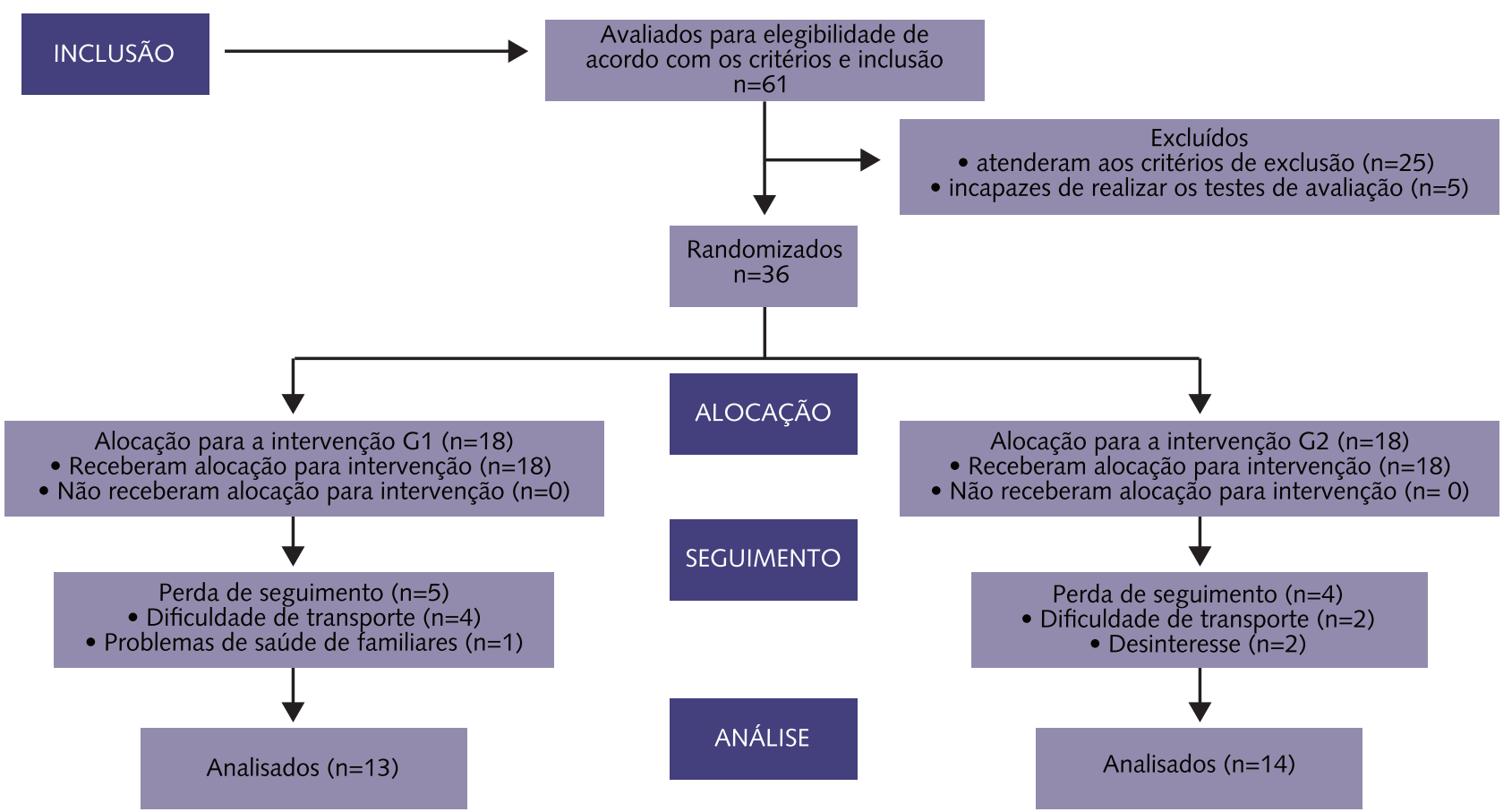

Figura 1 - Acompanhamento e perda amostral. 
A observação relacionada à frequência de participação nos permite evidenciar que no G1, apenas 1 indivíduo frequentou entre 1 a 10 sessões, 4 participantes tiveram uma frequência entre 11 a 20 sessões, e 8 voluntários participaram entre 21 e 30 sessões. Enquanto que no G2 apenas 2 participantes frequentaram entre 1 a 10 sessões, 6 voluntários estiveram presentes entre 11 a 20 sessões e, 6 indivíduos participaram entre 21 a 30 sessões. Dessa forma, que a assiduidade nas sessões foi equilibrada (frequência média: $G 1=15,6 \pm 10,7$ vs. $G 2=16,5 \pm 9,5 ; p=0,425$ ). A adesão relativamente baixa ao programa ocorreu principalmente devido às dificuldades de acesso ao local de prática e problemas pessoais de saúde.

\section{Efeitos dos programas de exercícios sobre a mobilidade funcional}

Após a aplicação do programa de exercícios, ao analisar o efeito da intervenção intragrupos, observa-se que ambos os grupos apresentaram melhora significativa nos parâmetros neuromusculares avaliados no STS-5, SMW e FRT, e que estes grupos não foram estatisticamente diferentes quando comparados entre si. A comparação dos Grupos 1 e 2 antes e após 4 meses de intervenção evidenciou os resultados expressos na Tabela 2.

Tabela 2 - Parâmetros neuromusculares do Grupo Fortalecimento e Mobilidade funcional (G1) e Grupo Alongamento e Mobilidade Articular (G2) na avaliação inicial e após 4 meses de intervenção.

\begin{tabular}{llccc}
\hline & Grupos & M1 & $M 2$ & Valor $p$ \\
\hline Teste STS-5(seg) & G1 & $12,62(3,49)$ & $8,39(1,27)$ & $0,001^{*}$ \\
& G2 & $12,20(2,57)$ & $8,05(1,32)$ & $0,000^{*}$ \\
\hline Teste SMW (seg) & G1 & $4,41(1,23)$ & $3,90(0,77)$ & $0,014^{*}$ \\
& G2 & $4,22(0,48)$ & $3,59(0,41)$ & $0,000^{*}$ \\
\hline \multirow{2}{*}{ Teste FRT (cm) } & G1 & $26,38(4,85)$ & $31,61(5,02)$ & $0,001^{*}$ \\
& G2 & $24,57(4,83)$ & $30,34(4,33)$ & $0,001^{*}$ \\
\hline
\end{tabular}

M1 = Média (DP) na avaliação inicial; $M 2=$ Média (DP) após 4 meses de intervenção. ${ }^{*} p<0,05$. M1 vs. M2 Teste t de Student para amostras pareadas G1 vs. G2 Teste t de Student para amostras independentes.

\section{DISCUSSÃO}

Os achados de nosso estudo evidenciam que os exercícios para fortalecimento e coordenação bem como os alongamentos realizados foram capazes de promover benefícios na mobilidade funcional de idosas com baixa massa óssea, verificados através dos testes SMW, STS-5 e FRT. De fato Kato-Narita et al2 defendem que diferentes abordagens devam ser utilizadas para prevenir quedas e restaurar habilidades funcionais, sem assumir que apenas o treino de equilibrio irá necessariamente promover benefícios.

Dessa forma, o exercício vem sendo reconhecido na literatura como um método eficaz no combate à osteoporose, sendo capaz de reduzir o risco de quedas através da melhora da força, qualidade da marcha e aumento da massa óssea ${ }^{12}$. Além disso, ele melhora o equilíbrio que é essencial para a realização de atividades diárias e qualidade de vida ${ }^{21}$.

Porém, não há consenso na literatura vigente sobre a influência dos alongamentos e dos exercícios de equilíbrio e mobilidade funcional em idosos ${ }^{15}$, sendo importante determinar seus efeitos. Para tanto, utilizamos exercícios que envolviam treinamento de força, resistência e flexibilidade de intensidade progressiva, com desafios de equilíbrio, marcha e coordenação motora, associado à educação 
contra o risco de quedas e orientações domiciliares, como proposto por Blank et $a R^{2}$. No entanto, realizamos tarefas distintas nos diferentes grupos estudados.

A partir disso, encontramos uma melhora nos resultados do STS-5 e no SMW. Os mesmos testes foram utilizados para a avaliação da mobilidade funcional em um estudo realizado por Sherrington $e^{2} a^{23}$, que submeteram 85 idosos a um programa de 14 exercícios durante 5 semanas, obtendo ganhos significativamente maiores que no grupo controle, que não sofreu intervenção. Desta forma, estes pesquisadores evidenciaram um progresso no STS-5 e uma caminhada mais rápida no SMW, muito embora não tenha sido expressada diferença significativa na reavaliação de força e equilíbrio estático.

A melhora encontrada em nossos resultados, após a intervenção, sem diferença significativa entre os grupos estudados, corrobora com análise feita por Liddle $e a^{24}$, que identificaram que quando os grupos experimental e controle recebem programas supervisionado de exercício de conteúdo variável, ambos atingem resultados positivos. É válido considerar, no entanto, que os testes utilizados não tenham sido suficientemente sensíveis para identificar possíveis variações entre a evolução dos dois grupos no presente estudo. De modo geral, progressos na mobilidade funcional através de um programa de exercícios de equilíbrio e treino resistido são observados exclusivamente no grupo experimental quando o grupo controle não sofre intervenções e mantém seu estilo de vida sedentário ${ }^{7,12}$.

Análises dos efeitos secundários à atividade física revelam que o exercício induz benefícios específicos ao modo de treinamento aplicado ${ }^{25}$. O que nos leva a concluir que os resultados após um programa de exercícios são proporcionais aos tipos de atividades desenvolvidas. Por isso, é provável que tenham ocorrido ganhos na mobilidade funcional dos indivíduos de ambos os grupos deste estudo pelo fato dos exercícios propostos, promoverem estímulos proprioceptivos, mudanças de posicionamento e variações posturais.

É interessante observar ainda o estudo de Alfieri et al ${ }^{11}$ que observou melhora significativa da mobilidade funcional apenas no grupo que realizou atividades envolvendo estímulos multissensoriais, enquanto que o grupo que realizou apenas exercícios de fortalecimento não obteve melhoras. É possível que pelo fato das atividades de fortalecimento terem sido desenvolvidas na posição sentada, elas tenham eliminado os estímulos proprioceptivos, não gerando as melhoras avaliadas. O presente estudo, por outro lado, utilizou posicionamentos diversificados em ambos os grupos, não se restringindo apenas à posição sentada, o que pode justificar que a estimulação dos sistemas visual e vestibular possa fundamentar a melhora significativa dos parâmetros avaliados.

Além desses achados, é válido ainda considerar outros efeitos do exercício, mesmo que não tenham sido objetivos de avaliação deste estudo. É comprovado que existe significância na relação entre a saúde percebida de pessoas e o seu desempenho físico - força muscular, velocidade de caminhada e equilíbrio ${ }^{26}$. Além disso, tem sido defendido que o exercício físico é benéfico para o combate à dor lombar, melhora da qualidade de vida e prevenção do risco de quedas ${ }^{27,28}$. Tais considerações evidenciam que diversos outros fatores estão envolvidos no desempenho físico de indivíduos, o que nos obriga a considerar que possíveis reduções nos níveis de dor e maior motivação pessoal podem justificar a melhora verificada no grupo de alongamento. Tanto que Varejão $e t$ a ${ }^{29}$ identificaram benefícios na autonomia de idosos após programas de alongamentos implementados durante 24 semanas.

Desta forma, programas de fortalecimento e estímulos proprioceptivos, bem como os alongamentos, são tidos como métodos comuns de intervenção, entre- 
tanto mais estudos necessitam comprovar os benefícios de formas de intervenção diversificadas ${ }^{23}$. Assim, como regra geral é aconselhável evitar a restrição de mobilidade, pois isso poderá piorar o padrão motor, equilíbrio e força, aumentando o risco de lesões e quedas ${ }^{20}$.

Para finalizar, é possível que este estudo não tenha evidenciado diferenças significantes entre os resultados dos grupos avaliados devido ao curto tempo de intervenção. E pode ainda ser identificada como uma limitação do estudo, o número amostral reduzido devido à grande perda de voluntários durante a realização da pesquisa. Estes aspectos justificam a realização de mais estudos para analisar de forma mais consistente os benefícios promovidos pelo exercício físico de maneira específica.

\section{CONCLUSÃO}

Considerando a hipótese de que ocorram mais benefícios mediante a maior diversidade de atividades envolvidas em um programa de treinamento, os nossos resultados permitem concluir que os exercícios físicos de fortalecimento muscular, de equilíbrio e alongamentos são capazes de trazer benefícios para idosas com baixa massa óssea, através de melhora na sua mobilidade funcional.

\section{Contribuição dos autores}

A pesquisadora Mariana de Sousa Moura participou da parte experimental da pesquisa, tanto na aplicação dos testes quanto na implementação dos programas de exercícios propostos, foi responsável pela coleta e tabulação dos dados, desenvolveu a análise estatística e além disso realizou a pesquisa bibliográfica e redigiu o texto do artigo. Profa ${ }^{a}$. Dra ${ }^{a}$. Márcia Alessandra Carneiro Pedrosa contribuiu no processo de submissão da pesquisa ao Comitê de Ética e Fundação de Amparo à Pesquisa do Estado de Pernambuco, auxiliou na elaboração dos programas de exercícios, contribuiu na pesquisa bibliográfica e orientou a construção do texto e todo o trabalho científico. Os demais autores, Eduarda Lubambo Costa, Paulo Sérgio de Castro Bastos Filho, Larissa Bouwman Sayão e Tiago Siqueira de Sousa tiveram fundamental importância na aplicação dos testes de avaliação, na intervenção através dos programas de exercícios, bem como na coleta e tabulação dos dados.

\section{Agradecimentos/ financiamento}

A pesquisa foi financiada pela Fundação de Amparo à Pesquisa do Estado de Pernambuco - FACEPE.

Agradecemos a todos os participantes envolvidos na pesquisa, pesquisadores, professora orientadora, funcionários do setor de Reumatofisioterapia do Hospital das Clínicas - HC/UFPE, e especialmente aos voluntários, sem a dedicação e disponibilidade dos mesmos não teria sido possível o êxito do nosso estudo.

\section{REFERÊNCIAS}

1. Ministério da Saúde. SUS gasta 81 milhões com fraturas em idosos em 2009. Agência Saúde, 2009. Disponível em: <http://portal.saude.gov.br/portal/saude /visualizar_texto. cfm?idtxt=33674\&janela=1 >. Acessado em 29 de março de 2012.

2. World Health Organization. Assessment of fracture risk and its role in screening for postmenopausal osteoporosis. In WHO Technical Report Series. Geneva, Switzerland: World Health Organization; 843, 1994.

3. Bruyère O, Reginster JY, Croisier JL, Crielaard JM, Maquet D. Rehabilitation in Osteoporotic Subjects - Myth or Reality? European Musculoskeletal Review 2010;5(1):36-9. 
4. Smulders E, Lankveld W, Laan R, Duysens J, Weerdesteyn V. Does osteoporosis predispose falls? A study on obstacle avoidance and balance confidence. BMC Musculoskeletal Disorders 2011;12:1.

5. Garcia PA, Dias JMD, Dias RC, Santos P, Zampa CC. Estudo da relação entre função muscular, mobilidade funcional e nível de atividade física em idosos comunitários. Rev Bras Fisioter 2011;15(1):15-22.

6. Sagiv M. The role of physical activity in the elderly as primary prevention. Eur Rev Aging Phys Act 2007;4(2):59-60.

7. Granacher U, Lacroix A, Muehlbauer T, Roettger K, Gollhofer A. Effects of Core Instability Strength Training on Trunk Muscle Strength, Spinal Mobility, Dynamic Balance and Functional Mobility in Older Adults. Gerontology 2012, 24 de Outubro. Disponível em <www.karger.com/ger>

8. Fabrício SCC, Rodrigues RAP, Costa Júnior, M L. Causas e consequências de quedas de idosos atendidos em hospital público. Rev Saúde Pública 2004;38(1):93-99.

9. Peel NM, Kuys SS, Klein K. Gait Speed as a Measure in Geriatric Assessment in Clinical Settings: A Systematic Review. J Gerontol A Biol Sci Med Sci 2012,24 de Agosto; first published online.

10. Papaioannou A, Adachi JD, Winergard K, et al. Efficacy of home-based exercise for improving quality of life among elderly women with symptomatic osteoporosis-related vertebral fractures. Osteoporos Int 2003;14(8):677-682.

11. Liu-ambrose T, Khan KM, Eng JJ, et al. Resistance and Agility Training Reduce Fall Risk in Women Aged 75 to 85 with Low Bone Mass: A 6-Month Randomized, Controlled Trial. J Am Geriatr Soc 2004;52(5):657-665.

12. Park H, Kim KJ, Komatsu T, Park SK, Mutoh Y. Effect of combined exercise training on bone, body balance, and gait ability: a randomized controlled study in community-dwelling elderly women. J Bone Miner Metab 2008;26:254-259.

13. Sherrington C, Whitney JW, Lord SR, et al. Effective Exercise for the Prevention of Falls: A Systematic Review and Meta-Analysis. JAGS 2008;56(12):2234-2243.

14. De Kam D, Smulders E, Weerdesteyn V, Smits-engelsman BC. Exercise interventions to reduce fall-related fractures and their risk factors in individuals with low bone density: a systematic review of randomized controlled trials. Osteoporos Int 2009;20(12):2111-25.

15. Orr R, Raymond J, Fiatarone SM. Efficacy of progressive resistance training on balance performance in older adults: a systematic review of randomized controlled trials. Sports Med 2008;38(4):317-43.

16. Liu-ambrose T, Ahamed Y, Graf P, Feldman F, Robinovitch SN. Older Fallers With Poor Working Memory Overestimate Their Postural Limits. Arch Phys Med Rehabil 2008;89(7):1335-1340.

17. Brucki SMD, Nitrini R, Caramelli P, et al. Sugestões para o uso do mini-exame do estado mental no Brasil. Arq. Neuro-Psiquiatr 2003;61(3B):777-781.

18. Tiedmann A, Shimada H, Sherrington C, Murray S, Lord SR. The comparative ability of eight functional mobility tests for predicting falls in community-dwelling older people. Age And Ageing 2008;37:430-435.

19. Figueiredo KM, Lima KC, Guerra RO. Instrumentos de Avaliação do Equilíbrio Corporal em Idosos. Rev Bras Cineantropom Desempenho Hum 2007;9(4):408-413.

20. Kato-narita EM, Nitrini R, Radanovic M. Assessment of balance in mild and moderate stages of Alzheimer's disease, Implications on falls and functional capacity. Arq Neuropsiquiatr 2011;69(2-A):202-207.

21. Alfieri FM, Riberto M, Gatz LS, et al. Functional mobility and balance in community-dwelling elderly submitted to multisensory versus strength exercises. Clinical Interventions in Aging 2010;5:181-185.

22. Blank WA, Freiberger E, Siegrist M, et al. An interdisciplinary intervention to prevent falls in community-dwelling elderly persons: protocol of a cluster-randomized trial [PreFalls] BMC Geriatrics 2011, 11:7.

23. Sherrington C, Pamphlett PI, Jacka JA, et al . Group exercise can improve participants' mobility in an outpatient rehabilitation setting: a randomized controlled trial. Clin Rehabil 2008;22(6):493-502. 
24. Liddle SD, Baxter GD, Gracey JH. Exercise and chronic low back pain: what works? Pain 2004;107:176-190.

25. Lee MJ, Kilbreath SL, Singh MF, et al. Comparison of Effect of Aerobic Cycle Training and Progressive Resistance Training on Walking Ability After Stroke: A Randomized Sham Exercise-Controlled Study. J Am Geriatr Soc 2008;56(6):976-985.

26. Ferreira FFP, Izzo H, Filho WJ. Impacto da capacidade física na saúde percebida entre idosos em velhice avançada. Saúde coletiva 2007;4(17):154-157.

27. Liu-ambrose TYL, Khan KM, Eng JJ, Lord SR, Mckay BLHA. Both resistance and agility training reduce back pain and improve health-related quality of life in older women with low bone mass. Osteoporos Int 2005;16:1321-1329.

28. Hubbard RE, Fallah N, Searle SD, Mitnitski A, Rockwood K. Impact of Exercise in Community-Dwelling Older Adults. PLoS ONE 2009;4(7):6174.

29. Varejao RV, Dantas EHM, Matsudo SMM. Comparação dos efeitos do alongamento e do flexionamento, ambos passivos, sobre os níveis de flexibilidade, capacidade funcional e qualidade de vida do idoso. R bras Ci e Mov 2007; 15(2): 87-95.

$$
\begin{array}{r}
\text { Endereço para Correspondência } \\
\text { Mariana de Sousa Moura } \\
\text { R. Prof. Augusto Lins e Silva, 668/902, } \\
\text { Boa Viagem, Recife, PE, } \\
\text { CEP: 51130-030. } \\
\text { E-mail: marimoura10@hotmail.com . } \\
\text { (81) } 33413137 \text { / (81) } 97076137
\end{array}
$$

Recebido $07 / 12 / 2012$

Revisado 07/12/2012

$23 / 01 / 2013$ 